\title{
Energy Resolution Contributions in Reach-Through Avalanche Photodiodes
}

\author{
A. L. Gouvea, Student Member, IEEE, and L. M. P. Fernandes
}

\begin{abstract}
Avalanche photodiodes (APD) are used in the muonic helium Lamb shift experiment for detection of $8 \mathrm{keV}$ X-rays. Reach-through APDs from Hamamatsu Photonics have been investigated for $\mathrm{X}$-ray detection as alternative to conventional APDs. In order to evaluate the different contributions to the energy resolution obtained in a reach-through APD, its response to both $8 \mathrm{keV} \mathrm{X}$-rays and visible light pulses was investigated. The intrinsic resolution was estimated by further measurements of the electronic noise contribution and the gain non-uniformity was assessed. The excess noise factor was determined. Results at different temperatures are presented.
\end{abstract}

Index Terms-Avalanche photodiodes, electronic noise, energy resolution, excess noise factor, gain non-uniformity, intrinsic resolution, light detection, reach-through avalanche photodiodes, $\mathrm{X}$-ray detectors.

\section{INTRODUCTION}

A VALANCHE PHOTODIODES (APD) have shown attractive properties in many applications, such as medical imaging, nuclear and particle physics, as photosensors coupled to scintillators and as soft X-ray detectors [1], [2]. A particular application of large area APDs is the muonic helium Lamb shift experiment [3], where they are used for detection of $8 \mathrm{keV}$ $\mathrm{X}$-rays.

Reach-through APDs (RT-APD) from Hamamatsu Photonics, with thicker depletion layer (about $130 \mu \mathrm{m}$ ), are in principle more suitable for the detection of $8 \mathrm{keV} \mathrm{X}$-rays ([4], [5], [6]) compared to traditional APDs [7], [8], [9]. This is due to their superior detection efficiency, which may be above $90 \%$ for $8 \mathrm{keV} \mathrm{X-rays} \mathrm{[6].} \mathrm{This} \mathrm{new} \mathrm{type} \mathrm{of} \mathrm{APD,} \mathrm{with} \mathrm{circular}$ shape and $3 \mathrm{~mm}$ diameter, has shown excellent properties in $\mathrm{X}$-ray detection operating at room temperature [4], [5]. A new design was later introduced by Hamamatsu, a transmission type reach-through APD made to detect X-rays from one side and $\gamma$-rays from the other side using a $\mathrm{CsI}(\mathrm{Tl})$ crystal coupled to the APD [6]. The performance of this type of APD has been investigated for the detection of $8 \mathrm{keV} \mathrm{X-rays} \mathrm{in} \mathrm{order} \mathrm{to} \mathrm{evaluate}$

\footnotetext{
Manuscript received February 26, 2014; revised July 20, 2014; accepted September 01, 2014. Date of publication September 24, 2014; date of current version October 09, 2014. This work was supported by FEDER through COMPETE and by National funds through FCT in the frame of project PTDC/FIS/102110/2008. A. L. Gouvea acknowledges grant from FCT (RSFRH/BD/66731/2009).

The authors are with the Instrumentation Center, University of Coimbra, P-3004-516 Coimbra, Portugal (e-mail: andrea.gouvea@gian.fis.uc.pt).

Color versions of one or more of the figures in this paper are available online at $\mathrm{http}: / /$ ieeexplore.ieee.org.

Digital Object Identifier 10.1109/TNS.2014.2356854
}

its suitability for application in the muonic helium Lamb Shift Experiment [10]. Two prototypes with $5 \times 5$ and $3 \times 5 \mathrm{~mm}^{2}$ active areas were investigated. The operational parameters, such as gain, gain non-linearity, minimum detectable energy and energy resolution were determined as a function of bias voltage and temperature. The $5 \times 5$ prototype turned out to have the best performance. The best energy resolution achieved was $9.5 \%$ at $0^{\circ} \mathrm{C}$, with a corresponding minimum detectable energy of $0.25 \mathrm{keV}[10]$.

Since the energy resolution obtained is a critical parameter for the experiment, and in order to understand its behavior, the different contributions were evaluated. In general, the energy resolution is defined by the FWHM of the peak obtained in the amplitude spectrum. Several contributions are responsible for the peak broadening in silicon APDs:

- statistical fluctuations arising from the discrete nature of the number of electron-hole pairs produced in silicon and to the avalanche process;

- gain non-uniformity due to impurities and crystal imperfections;

- electronic noise, generated by the detector dark current and by the preamplifier.

The energy resolution of a measured peak $(\Delta \mathrm{E})$, can be mathematically represented as the quadratic addition of the three contributions:

$$
(\Delta \mathrm{E})^{2}=\left(\Delta \mathrm{E}_{\mathrm{s}}\right)^{2}+\left(\Delta \mathrm{E}_{\mathrm{u}}\right)^{2}+\left(\Delta \mathrm{E}_{\mathrm{n}}\right)^{2}
$$

where $\Delta \mathrm{E}_{\mathrm{s}}$ corresponds to the statistical fluctuations, $\Delta \mathrm{E}_{\mathrm{u}}$ the fluctuations associated to the gain non-uniformity and $\Delta \mathrm{E}_{\mathrm{n}}$ the noise fluctuations [7], [11].

The intrinsic energy resolution, which is given by all contributions except noise, is essentially defined by the fluctuations associated to the production of electron-hole pairs and to the electron multiplication process. The spatial non-uniformity may also contribute to the intrinsic energy resolution, particularly in X-ray detection, due to the point-like absorption of the $\mathrm{X}$-ray, while in light detection the primary electron-hole pairs are spread over the APD volume, if the whole area is illuminated, averaging local gain variations [8], [9], [12]. Non-uniform silicon resistivity leads to electric field variations, and consequently to gain fluctuations. Therefore the contributions to take into account in the intrinsic energy resolution determination are different for light and X-ray detection. Another relevant aspect to take into account is the Fano factor, $\mathrm{f}$, which describes the relative variance in the process of electron-hole pair production for energy loss in matter. This parameter is well known in the area of radiation detectors, being in silicon equal to 0.12 


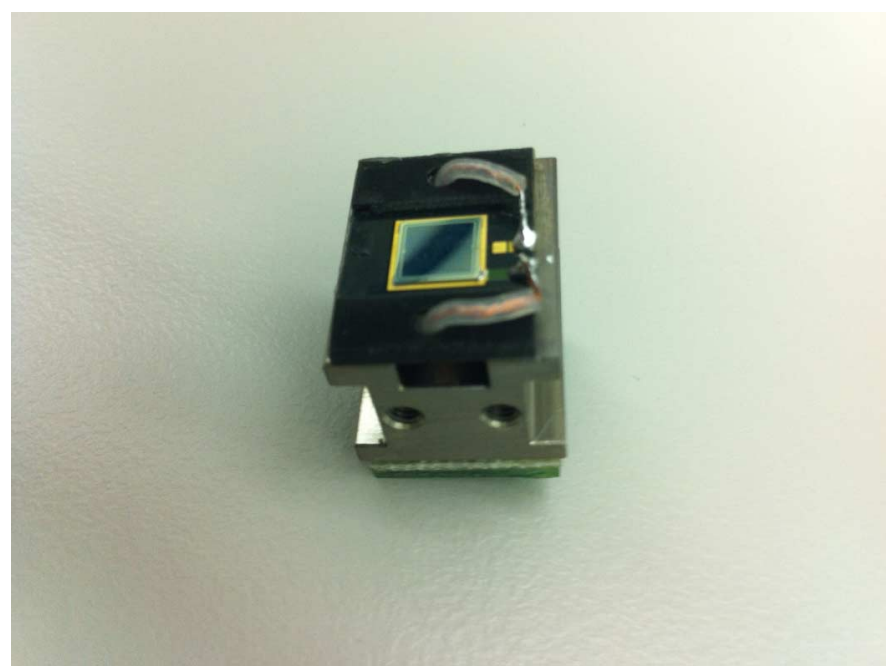

Fig. 1. Photograph of the reach-through APD used in this work, mounted on a titanium piece for efficient cooling.

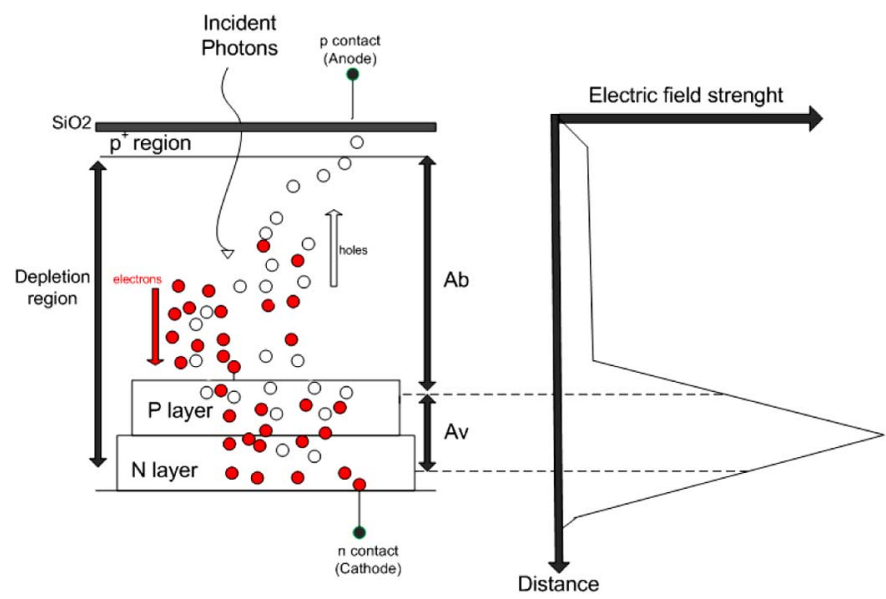

Fig. 2. Schematic of RT-APD. The structure and electric field strength in the different regions, $\mathrm{Ab}$ is the absorption region and $\mathrm{Av}$ is the avalanche or multiplication region. The depletion region is composed by both the absorption and avalanche regions. The circles represent charge carriers, electrons and holes, and the arrows the corresponding direction towards the collection electrodes.

at $77 \mathrm{~K}$ [11]. Monte Carlo simulation showed this parameter is almost constant with temperature presenting small fluctuations around 0.117 [13].

\section{EXPERIMENTAL SETUP AND METHOD}

Fig. 1 shows a photograph of the reach-through APD used for this study. It is a $5 \times 5 \mathrm{~mm}^{2}$ prototype, model $\mathrm{S} 10936$ 0374 from Hamamatsu Photonics. According to the manufacturer datasheet, the breakdown voltage is $623 \mathrm{~V}$ at room temperature $\left(25^{\circ} \mathrm{C}\right)$ and the operating conditions for a gain of 39 are $200 \mathrm{~V}$ bias voltage, $8 \mathrm{nA}$ dark current and $22 \mathrm{pF}$ capacitance.

As shown in Fig. 2, in the front surface of the detector, there is a $\mathrm{SiO}_{2}$ layer, which is used to prevent the detector from high dark currents. The absorption region $(\mathrm{Ab})$ is located a few $\mu \mathrm{m}$ below. This is where most of the photons interact with Si material, producing a fast photoelectron, which slows down

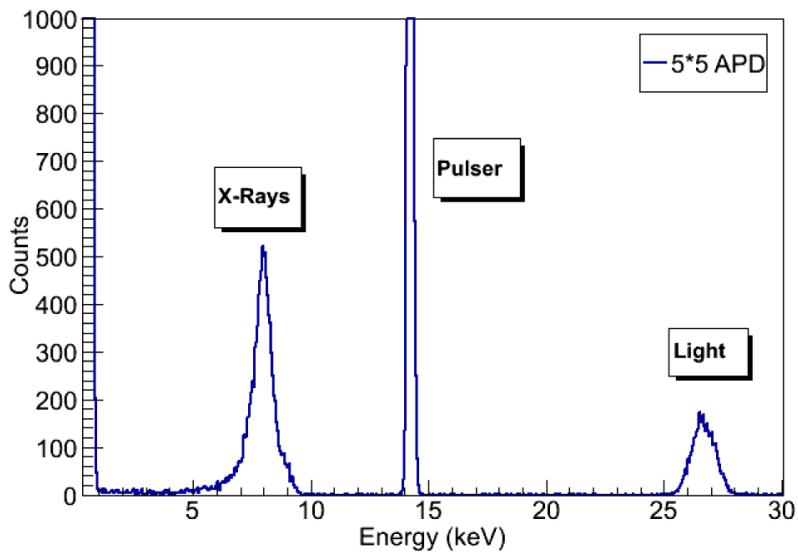

Fig. 3. Typical pulse-height distribution obtained in the RT-APD from $8 \mathrm{keV}$ $\mathrm{X}$-rays emitted by a Zn-65 source, visible light pulses from a LED and reference pulses directly injected in the preamplifier test input.

in successive collisions, resulting in a number of electron-hole pairs (in average, one per $3.62 \mathrm{eV}$ of energy deposited). This zone is characterized by a low electric field, which increases slowly with depth, being responsible for separating the holes and electrons generated, each of them moving towards the respective electrode. However, before the collection, the electrons will cross an intense electric field zone, the avalanche region (Av), where the charge will be multiplied in sucessive collisions by impact ionization [11]. The average absorption length for incident X-rays depends strongly on the photon energy. For instance, for $2 \mathrm{keV}$ and $6 \mathrm{keV}$ the average absorption lenght is $4 \mu \mathrm{m}$ and $30 \mu \mathrm{m}$, respectively [1], [7].

The RT-APD response has been investigated in a vacuum chamber, where it can be cooled down to about $-30^{\circ} \mathrm{C}$ and temperature controlled within $0.1^{\circ} \mathrm{C}$. A $\mathrm{Zn}-65$ radioactive source emitting $8 \mathrm{keV}$ X-rays was placed inside the chamber, together with a light guide connected to a LED. Both the radioactive source and the light guide were positioned in front of the RT-APD surface at about $1 \mathrm{~cm}$ distance. The LED was operated in pulse mode with peak emission at $600 \mathrm{~nm}$ and was supplied by a "LED pulser", giving pulses of 100 to $500 \mathrm{~ns}$ width and up to $-10 \mathrm{~V}$ amplitude. This way, the X-ray and visible light signals can be measured at the same time and compared. The signals from a RAL-108 A preamplifier [14] connected to the APD are driven to an ORTEC spectroscopy amplifier which in turn is connected to a Pocket MCA (AmpTek $8000 \mathrm{~A}$ ), where pulse-height distributions are recorded. A typical signal at the RAL preamplifier output has about 200 ns rise-time, more than $100 \mu$ s fall-time and amplitudes between 100-200 mV. Amplifier shaping time constants of $0.5 \mu$ s were used for both integration and differentiation.

Fig. 3 shows a typical pulse-height distribution obtained in the RT-APD for three different signals: $8 \mathrm{keV}$ X-rays, visible light pulses and test pulses from a pulse generator.

By simultaneous measurements of X-rays and visible light pulses detected in the APD, and also test pulses from a reference pulser, the contributions to the energy resolution, expressed in equation (1) can be determined from the width of each peak in the pulse-height distribution. The centroid of the X-ray peak defines, the energy calibration for the visible light peak [10]. 
The electronic noise contribution to the energy resolution is determined by the pulser peak broadening in the pulse-height distribution (FWHM in units of energy), seen in Fig 3. The position of the reference pulse peak does not depend on the detector gain [9], [12]. The noise contribution can be subtracted from the total energy resolution to extract the intrinsic energy resolution of the detector and also to determine the excess noise factor.

\section{Contributions to the Energy Resolution}

The signal variance associated to the statistical contributions in equation (1) is given by:

$$
\sigma_{\mathrm{s}}^{2}=\sigma_{\mathrm{N}}^{2}+\mathrm{N}(\mathrm{F}-1)
$$

where $\mathrm{N}$ is the number of primary electrons, $\sigma_{\mathrm{N}}$ its variance and $\mathrm{F}$ the excess noise factor. $\mathrm{F}$ is related to the variance of the gain during one avalanche, $\sigma_{\mathrm{A}}^{2}$, for a specific gain $\mathrm{G}$. It is defined by:

$$
\mathrm{F}=1+\frac{\sigma_{\mathrm{A}}^{2}}{\mathrm{G}^{2}}
$$

In light detection, if the whole detector active area is irradiated, the contribution associated to gain non-uniformity in equation (1) is negligible since the final pulse results from the average response to the full amount of photons interacting in the absorption region of the APD. The intrinsic energy resolution for light detection, considering the variance of the number of primary electrons described by Poisson statistics $\left(\sigma_{\mathrm{N}}^{2}=\mathrm{N}\right)$, is giving by:

$$
R_{\mathrm{int}}^{\mathrm{L}}=2.355 \frac{\sigma_{\mathrm{s}}}{\mathrm{N}}=2.355 \sqrt{\frac{\mathrm{F}}{\mathrm{N}}}
$$

where $\sigma_{s}^{2}=\mathrm{NF}$.

In X-ray detection, one needs to take into account the nonuniformity contribution and the Fano factor, $\mathrm{f}$. Hence, the variance in the number of primary electrons is $\sigma_{\mathrm{N}}^{2}=\mathrm{Nf}$ and the statistical contribution takes the form:

$$
\sigma_{\mathrm{s}}^{2}=\mathrm{N}(\mathrm{F}+\mathrm{f}-1)
$$

The intrinsic energy resolution in X-ray radiation will be:

$$
\mathrm{R}_{\mathrm{int}}^{\mathrm{X}}=2.355 \sqrt{\frac{\mathrm{F}+\mathrm{f}-1}{\mathrm{~N}}+\left(\frac{\sigma_{\mathrm{U}}}{\mathrm{G}}\right)^{2}}
$$

where $\sigma_{\mathrm{U}} / \mathrm{G}$ is the relative standard deviation associated to gain non-uniformities.

If the number of primary electrons produced in silicon by both X-ray and light pulse is the same, from equations (4) and (6), the intrinsic energy resolution for X-rays and light in the pulse-height distributions are related by:

$\left(\mathrm{R}_{\text {int }}^{\mathrm{X}}\right)^{2}-\left(\mathrm{R}_{\text {int }}^{\mathrm{L}}\right)^{2}=(2.355)^{2}\left[\left(\frac{\mathrm{F}+\mathrm{f}-1}{\mathrm{~N}}\right)+\left(\frac{\sigma_{\mathrm{u}}}{\mathrm{G}}\right)^{2}-\left(\frac{\mathrm{F}}{\mathrm{N}}\right)\right]$

Fig. 4 shows the energy resolution obtained for $8 \mathrm{keV} \mathrm{X-rays}$ and visible light pulses with the same pulse height, as a function of gain for different temperatures $\left(20^{\circ} \mathrm{C}, 0^{\circ} \mathrm{C}\right.$ and $\left.-20^{\circ} \mathrm{C}\right)$. The energy resolution improves with decreasing temperature for

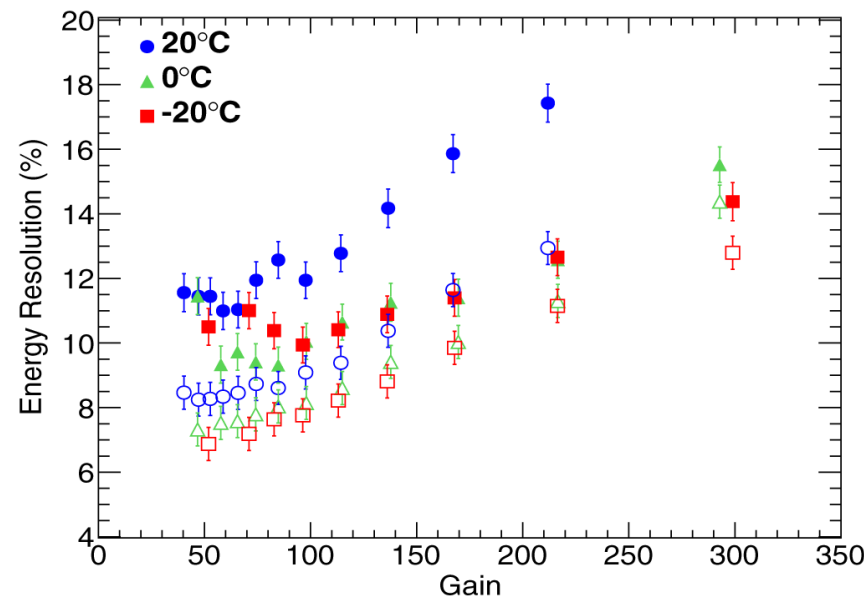

Fig. 4. Energy resolution for $8 \mathrm{keV} \mathrm{X-rays} \mathrm{(closed} \mathrm{symbols)} \mathrm{and} \mathrm{visible} \mathrm{light}$ pulses (open symbols) at different temperatures $\left(20^{\circ} \mathrm{C}, 0^{\circ} \mathrm{C}\right.$ and $\left.-20^{\circ} \mathrm{C}\right)$.

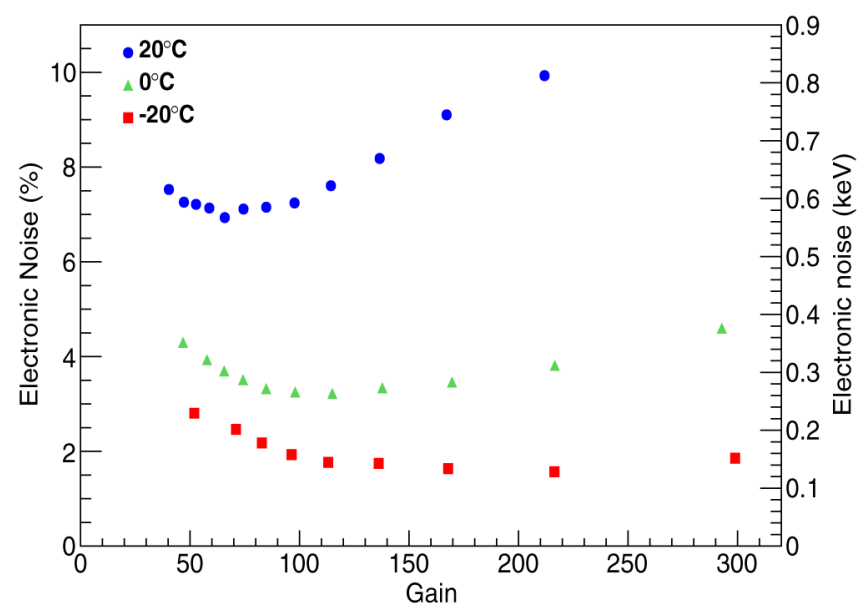

Fig. 5. Electronic noise contribution (FWHM) to the RT-APD energy resolution as a function of gain, for different temperatures, normalized to $8 \mathrm{keV}$ $\mathrm{X}$-rays.

both $\mathrm{X}$-rays and visible light, but the improvement is not significant from $0^{\circ} \mathrm{C}$ to $-20^{\circ} \mathrm{C}$. The optimum gain, providing the best energy resolution achieved, is in the region 50-100. As shown for X-rays, the energy resolution is better for lower gains and degrades for higher gains.

For both X-rays and visible light the energy resolution starts to increase slowly above a gain of 100 . This is related to the increasing dark current at higher temperatures and, in any case, to the excess noise factor.

\section{A. Electronic Noise}

Fig. 5 shows the electronic noise contribution (FWHM in units of energy and in \%) to the energy resolution as a function of gain for different temperatures $\left(-20^{\circ} \mathrm{C}, 0^{\circ} \mathrm{C}\right.$ and $\left.20^{\circ} \mathrm{C}\right)$. To express the noise contribution in units of energy, the position of the $8 \mathrm{keV} \mathrm{X}$-ray peak was taken for normalization. The electronic noise is strongly dependent on temperature, increasing for higher temperatures, and its variation with gain shows a minimum value which is $0.58 \mathrm{keV}(7 \%), 0.26 \mathrm{keV}(3.24 \%)$ and 
$0.13 \mathrm{keV}(1.5 \%)$ for $20^{\circ} \mathrm{C}, 0^{\circ} \mathrm{C}$ and $-20^{\circ} \mathrm{C}$, respectively. For higher gains, the electronic noise increase is more pronounced for higher temperatures, as a result of the larger dark current. The behavior of the noise contribution of the RT-APD with gain and with temperature is coherent with the result reported in [9], [12].

\section{B. Intrinsic Energy Resolution and Gain Non-Uniformity}

The intrinsic energy resolution is a characteristic inherent to the APD. It is originated from statistical fluctuations associated to the number of electron-hole pairs produced in the silicon volume and to the avalanche process. In X-ray detection the gain non-uniformity in the avalanche region is also a major contribution to the intrinsic energy resolution.

Fig. 6 shows the intrinsic energy resolution for $8 \mathrm{keV} \mathrm{X}$-rays and visible light pulses as a function of gain obtained at $20^{\circ} \mathrm{C}$ (a), $0^{\circ} \mathrm{C}(\mathrm{b})$ and $-20^{\circ} \mathrm{C}$ (c) respectively, as well as the quadratic difference between the two resolution curves. The electronic noise contribution was quadratically subtracted from the total energy resolution (Fig. 4) to obtain the intrinsic energy resolution. As expected, the intrinsic energy resolution for light is better than for X-rays as a result of the gain non-uniformity contribution. Fig. 6 also presents the quadratic difference between the energy resolution curves for $8 \mathrm{keV} \mathrm{X-rays} \mathrm{and} \mathrm{visible} \mathrm{light}$ pulses. This difference is important to deduce the gain non-uniformity from eq. (7).

The gain non-uniformity can significantly degrade the energy resolution for X-rays. This contribution was obtained as a function of gain and for temperatures of $20^{\circ} \mathrm{C}, 0^{\circ} \mathrm{C}$ and $-20^{\circ} \mathrm{C}$, using eq. (7) and the intrinsic energy resolution values of Fig. 6. The result is presented in Fig. 7. The non-uniformity obtained, $\left(\sigma_{\mathrm{U}} / \mathrm{G}\right)$, is $2.2 \% \pm 0.4 \%$ in good agreement with the results reported in [7], [12]. Nonetheless it is a bit higher than the one reported in [5], of about $1.4 \%$.

The additional contribution of the non-uniformity leads to the energy resolution deterioration and is more pronounced for higher gains. Regarding light detection, there is also an energy resolution degradation with increasing gain. Nevertheless for RT-APDs this increase is more noticeable than the one reported in [8].

\section{EXCESS NOISE FACTOR (F)}

The excess noise factor (ENF) is an intrinsic characteristic of the APD [7], [8], [9], [12] and can be determined according to the equation:

$$
\Delta \mathrm{E}^{2}=\left(\Delta \mathrm{E}_{\mathrm{n}}\right)^{2}+(2.355)^{2} \mathrm{FE} \varepsilon
$$

where $\Delta \mathrm{E}$ is the energy broadening of the visible light peak (FWHM, in units of energy), $\Delta \mathrm{E}_{\mathrm{n}}$ is the corresponding noise contribution width (FWHM, in units of energy), $\varepsilon=3.62 \mathrm{eV}$ is the mean energy to produce an electron-hole pair in silicon and $\mathrm{E}$ is the energy deposited in silicon by a calibration radioactive source giving the same pulse height as the visible light pulses. Equation (8) results from eq. (4) since $N=E / \varepsilon$.

The excess noise factor has been determined using eq. (8) as a function of the APD gain for different temperatures $\left(-20^{\circ} \mathrm{C}\right.$, $0^{\circ} \mathrm{C}$ and $20^{\circ} \mathrm{C}$ ), as shown in Fig. 8. As expected, $\mathrm{F}$ does not
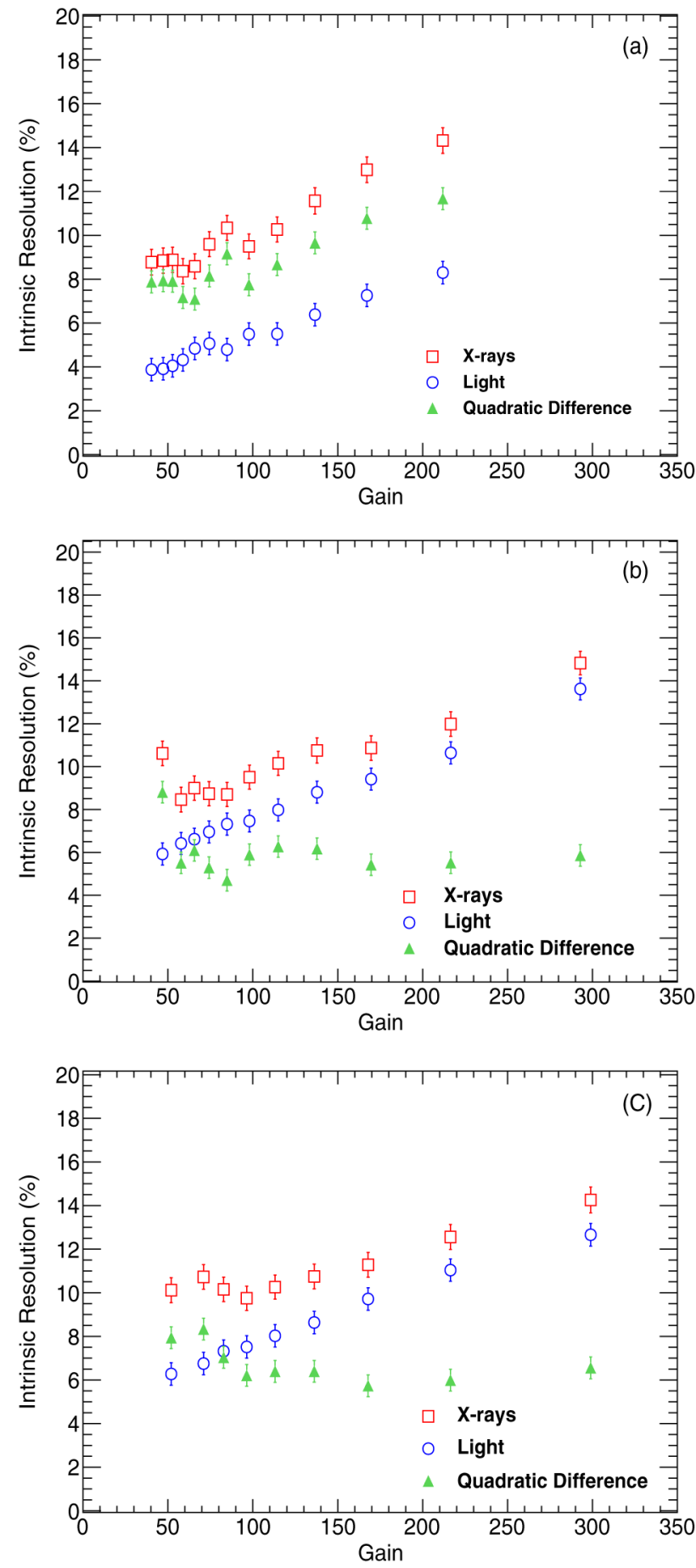

Fig. 6. Intrinsic energy resolution for $8 \mathrm{keV} \mathrm{X}$-rays and visible light pulses as a function of gain obtained at (a) $20^{\circ} \mathrm{C}$, (b) $0^{\circ} \mathrm{C}$ and (c) $-20^{\circ} \mathrm{C}$. The quadratic difference between the two curves is also showed.

depend on temperature and increases with gain [8], [9], [12]. It is about 2 for gains below 80 and about 3.2 for a gain of 200 . A linear fit to all measurements was made for gains above 50 . The linear dependence between $F$ and gain $(G)$ is expressed by:

$$
\mathrm{F}=0.009 \mathrm{G}+1.42
$$

Comparing these results with those from the LAAPDs from Radiation Monitoring Devices [7], for the same gain, $\mathrm{F}$ is almost 


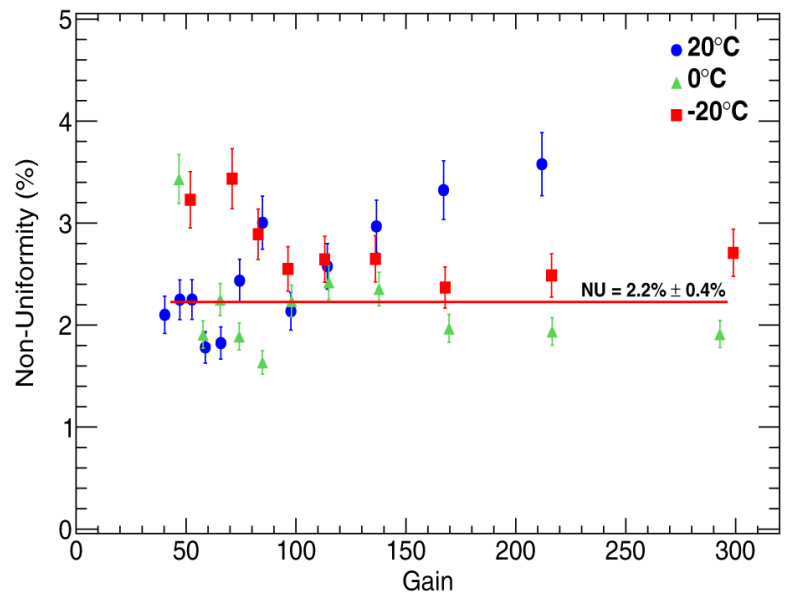

Fig. 7. Gain non-uniformity $\left(\sigma_{\mathrm{U}} / \mathrm{G}\right)$ obtained for three different temperatures, $20^{\circ} \mathrm{C}, 0^{\circ} \mathrm{C}$ and $-20^{\circ} \mathrm{C}$. The straight line shows the average off all points.

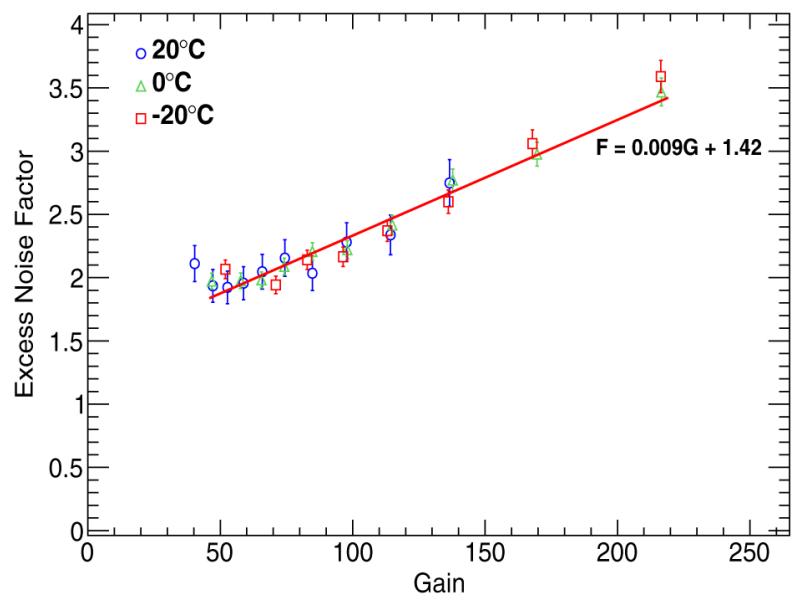

Fig. 8. Excess noise factor $(\mathrm{F})$ as a funtion of the APD gain $(\mathrm{G})$, for different temperatures. The straight line represents a fit to all measurements for gains above 50

twice higher for the RT-APD. For the same RT-APD structure, reported in [5], for a gain of 60 at $-20^{\circ} \mathrm{C}$ they obtained about 2.5 and our value is slightly lower, about 2 .

\section{CONCLUSIONS}

The contributions to the energy resolution obtained for $8 \mathrm{keV}$ $\mathrm{X}$-rays and visible light with reach-through avalanche photodiodes from Hamamatsu Photonics have been investigated through measurements with visible light pulses and test pulses directly injected in the preamplifier.

The comparison of the energy resolution obtained for both output signals with the same energy deposited in silicon makes it possible to determine the gain non-uniformity contribution, which affects the energy resolution for X-rays but not for visible light if the whole area of the APD is irradiated. The non-uniformity was assessed. The result obtained is estimated in $2.2 \%$ with a contribution to the energy resolution similar to the other ones. However, as the non-uniformity is highly dependent on the manufacturing process, differences between prototypes of the same series may be however significant.

The electronic noise contribution has been determined and has shown a strong dependence on temperature. This is mainly due to a dark current increase with temperature, which affects the overall APD performance at room temperature.

Moreover, the excess noise factor was determined as a function of gain for different temperatures. No significant dependence on temperature has been noticed. As a result, the energy resolution dependence on temperature cannot be attributed to the excess noise factor. On the other hand, the increase (linear dependence) of the excess noise factor with gain is one of the contributions to the energy resolution.

\section{ACKNOWLEDGMENT}

The authors would like to thank Paul Scherrer Institut, where this work was carried out.

\section{REFERENCES}

[1] D. Renker, "Properties of avalanche photodiodes for applications in high energy physics, astrophysics and medical imaging," Nucl. Instrum. Methods Phys. Res. A, vol. 486, pp. 164-169, 2002.

[2] L. M. P. Fernandes, J. A. M. Lopes, J. M. F. dos Santos, and C. A. N. Conde, "Application of large-area avalanche photodiodes to energydispersive x-ray fluorescence analysis," X-Ray Spectrom., vol. 30, pp. $164-169,2001$.

[3] A. Antognini, F. Biraben, J. M. R. Cardoso, D. S. Covita, A. Dax, and L. M. P. Fernandes et al., "Illuminating the proton radius conundrum: The mu He+ lamb shift," Can. J. Phys., vol. 89, pp. 47-57, 2011.

[4] J. Kataoka, T. Saito, Y. Kuramoto, T. Ikagawa, Y. Yatsu, and J. Kotoku et al., "Recent progress of avalanche photodiodes in high-resolution X-rays and $\gamma$-rays detection," Nucl. Instrum. Methods Phys. Res. A, vol. 541, pp. 398-404, 2005.

[5] Y. Yatsu, Y. Kuramoto, J. Kataoka, J. Kotoku, T. Saito, and T. Ikagawa et al., "Study of avalanche photodiodes for soft X-ray detection below 20 keV," Nucl. Instrum. Methods Phys. Res. A, vol. 564, pp. 134-143, 2006.

[6] S. Tanaka, J. Kataoka, Y. Kanai, Y. Yatsu, M. Arimoto, and M. Koizumi et al., "Development of wideband X-ray and gamma-ray spectrometer using transmission-type, large area APD," Nucl. Instrum. Methods Phys. Res. A, vol. 582, pp. 562-568, 2007.

[7] L. M. P. Fernandes, F. D. Amaro, A. Antognini, J. M. R. Cardoso, C. A. N. Conde, and O. Huot et al., "Characterization of large avalanche photodiodes in X-ray and VUV-light detection," J. Instrum., vol. 2, p. P08005, 2007.

[8] L. M. P. Fernandes, J. A. M. Lopes, J. M. F. dos Santos, P. E. Knowles, L. Ludhova, and F. Mulhauser et al., "LAAPD low temperature performance in X-ray and visible-light detection," IEEE Trans. Nucl. Sci., vol. 51, no. 4, pp. 1575-1580, Aug. 2004.

[9] L. Ludhova, F. D. Amaro, A. Antognini, F. Biraben, J. M. R. Cardoso, and C. A. N. Conde et al., "Planar LAAPDs: Temperature dependence, performance, and application in low-energy X-ray spectroscopy," Nucl. Instrum. Methods Phys. Res. A, vol. 540, pp. 169-179, 2005.

[10] A. L. Gouvea, A. Antognini, F. Kottmann, R. Pohl, and L. M. P. Fernandes, "Reach-through avalanche photodiodes in soft X-ray detection," IEEE Trans. Nucl. Sci., vol. 61, no. 4, pp. 2419-2424, Aug. 2014.

[11] G. F. Knoll, Radiation Detection and Measurements, 4th ed. Hoboken, NJ, USA: Wiley, 2010.

[12] M. Moszynski, M. Szawlowski, M. Kapusta, M. Balcerzyk, and D. Wolski, "Large area avalanche photodiodes in scintillation and X-rays detection," Nucl. Instrum. Methods Phys. Res. A, vol. 485, pp. 504-521, 2002.

[13] M. N. Mazziotta, "Electron-hole pair creation energy and fano factor temperature dependence in silicon," Nucl. Instrum. Methods Phys. Res. $A$, vol. 584, pp. 436-439, 2008.

[14] CLRC Rutherford Appleton Laboratory, Chilton, Didcot, Oxfordshire, OX11, 0QX, England. 